\title{
EL CARÁCTER MULTIMODAL DEL VERBO SENTIR: POLISEMIA Y TRANSITIVIDAD
}

\author{
MARLIES JANSEGERS, RENATA ENGHELS, IRASEMA CRUZ
}

\section{Abstract}

Within the domain of perception verbs, the Spanish sentir constitutes an interesting case because of its rich polysemy. The verb is not only used to denote several modalities of direct physical perception (tactile, olfactory, gustative and auditory), but it also extends towards the semantic domains of cognition and emotion. This article examines to what extent this multimodal character of the verb sentir influences on its degree of transitivity. It is well known that perception verbs in general do not respond to the definition of prototypical transitive verbs, given that the perception process represents a mental rather than a physical contact between the perceiver/subject and the stimulus/object. It is shown, however, that the verb sentir can be situated on different positions on the scale of transitivity, ranging from high transitivity to low transitivity, in function of the semantics it conveys. The validity of the elaborated hierarchy of transitivity is verified by means of empirical data.

Keywords: perception verbs, polysemy, transitivity, sentir

\section{Introducción}

Tradicionalmente, la transitividad ha sido definida como un concepto que tiene dos caras: una sintáctica y otra semántica. Sintácticamente, la alusión al esquema transitivo remite a un evento con dos participantes obligatorios; semánticamente, las cláusulas transitivas representan procesos en los que un participante agentivo ejerce una acción sobre un paciente, lo cual provoca una afectación en este último a causa de la transferencia de energía (Cano Aguilar 1981; Langacker 1991; García Miguel 1995, 2005; Taylor 1995, 2006). Estas dos caras de la transitividad han sido correlacionadas en el sentido de que el esquema semántico agente-acción-paciente ha sido proyectado sobre el esquema sintáctico $S$ - $V$ - $O$.

Sin embargo, varios autores (entre otros Cano Aguilar 1981; García-Miguel 1995; Taylor 1995) han señalado que esta correlación no es perfecta. En una oración como John resembles his brother (Taylor 1995: 209), no hay un agente que cause una transmisión de energía, por lo que el objeto directo (OD) tampoco se ve afectado. Por consiguiente, de acuerdo con Geisler (1989) conviene diferenciar bien entre la transitividad sintáctica y la transitividad semántica: mientras que aquella se caracteriza en términos discretos, esta se caracteriza por distintos grados de prototipicidad.

Hopper y Thompson (1980) definen la transitividad como un fenómeno gradual y multifactorial, distinguiendo unos parámetros en torno a sus elementos constitutivos. Por consiguiente, varios autores empezaron a reconocer el carácter gradual de esta noción postulando también una escala de agentividad (Dowty 1991; Langacker 1991). La noción de sujeto-agente prototípico se identifica tradicionalmente mediante una gama de rasgos semánticos como [+ control], [+ voluntad], [+ causa], [+ animacidad], [+ responsabilidad], [+ intención] e [+ iniciador de proceso]. Al contrario, la noción de paciente se relaciona prototípicamente con los rasgos opuestos de [- control], [- voluntad], [- causa], [- animacidad], 
[- responsabilidad], [- intención] e [- iniciador de proceso] (Langacker 1991; García-Miguel 1995; Van Valin y Lapolla 1997). De ahí resalta pues el carácter asimétrico de la transitividad semántica así como la posibilidad de reconocer sujetos (no controladores), objetos (no afectados) y verbos (que no codifican una transferencia de energía) más periféricos. En suma, los rasgos semánticos son nociones graduales que se ubican en un continuo. En esta discusión alrededor de la transitividad prototípica, los verbos de percepción (VdP) merecen una atención especial.

En la literatura sobre los VdP las relaciones entre los distintos verbos suelen describirse mediante oposiciones binarias. De esta forma, la naturaleza semántica de los VdP ha sido descrita tradicionalmente mediante dos parámetros, a saber la agentividad del perceptor y la modalidad específica de percepción. ${ }^{1}$ En español, esta clasificación semántica arroja el consabido diagrama siguiente (cf. entre otros Ibarretxe 1999: 50; Enghels 2007: 4):

\begin{tabular}{|l|l|l|}
\hline MODALIDAD & VOLUNTARIA & INVOLUNTARIA \\
\hline VISUAL & ver & mirar \\
\hline AUDITIVA & oír & escuchar \\
\hline TÁCTIL & sentir & tocar \\
\hline OLFATIVA & oler & olfatear $/$ husmear $/$ oler \\
\hline GUSTATIVA & $\ldots$ (notar) & $\ldots$ (probar) \\
\hline
\end{tabular}

Tabla 1. El paradigma básico de los VdP en español

Más concretamente, en lo que concierne al grado de agentividad del perceptor, se distingue entre VdP voluntaria y VdP involuntaria: los primeros tienen un perceptor/sujeto que se orienta activamente hacia el estímulo para obtener información, mientras que el sujeto de los VdP involuntaria experimenta el proceso de percepción de manera más bien pasiva, en el sentido de que el estímulo se impone a la consciencia del experimentante (cf. entre otros Ibarretxe 1999; Viberg 2005; Enghels 2007, 2013). Aparte de esta oposición, la semántica de cada verbo también varía según la modalidad de percepción (visual, auditiva, táctil, olfativa y gustativa). En suma, la idea según la cual los verbos formarían pares verbales como ver/mirar, oír/escuchar, sentir/tocar, oler/olfatear que representan una oposición léxica entre un término de carácter inagentivo (ver, oír, sentir, oler) frente a otro activo (mirar, escuchar, tocar, olfatear), ha sido muy defendida. ${ }^{2}$

Sintácticamente, la intrincada naturaleza del acto de percepción se traduce en una amplia gama de esquemas construccionales y complementos posibles (cf. García-Miguel 2005). Pueden entrar tanto en un esquema transitivo, ditransitivo como intransitivo. Además, en el esquema transitivo la variedad de complementos es notable, sobre todo con los $\mathrm{VdP}$ involuntaria ver y oír (p.ej. Enghels 2007, Hanegreefs 2008). Los VdP voluntaria mirar y escuchar y los verbos de las otras modalidades de percepción resultan mucho menos productivos desde el punto de vista sintáctico. Esto sugiere pues que dentro de la categoría de los verbos de percepción, no todos los miembros se comportan de la misma forma.

De la descripción que precede se deduce que la transitividad de los VdP no corresponde a la definición de la transitividad prototípica, especialmente en cuanto a la agentividad y el

\footnotetext{
${ }^{1}$ En este artículo, nos referimos a esta oposición de agentividad mediante los términos 'percepción voluntaria' vs. 'involuntaria', pero la misma variación ha sido definida también mediante otros términos, como por ejemplo percepción agentiva vs. no agentiva (Gruber 1967) o activa vs. pasiva (Willems 1983).

${ }^{2}$ La defienden por ejemplo Gruber (1967) y Scovel (1971).
} 
control se refiere. En primer lugar, los VdP no siempre transmiten energía de un agente a un paciente, dado que se encargan ante todo de codificar información proveniente del mundo externo (Ibarretxe 1999). Luego, el objeto de un VdP no es un paciente que sufra cambio de estado interno sino un estímulo percibido que le modifica al perceptor del evento mediante la información que le proporciona. Esto es, se trata de un objeto efectuado, más que afectado. Por consiguiente, los VdP han sido caracterizados tradicionalmente como verbos transitivos atípicos (Geisler 1989: 26; García-Miguel 1995: 73; Taylor 1995: 208-209; Enghels 2007, 2013).

No obstante, más recientemente, varios autores han aducido que incluso dentro del grupo semántico de los verbos de percepción, resulta necesario distinguir varios grados de agentividad. Así por ejemplo, Enghels (2013) propone una escala gradual de transitividad, donde los sujetos de percepción visual voluntaria se encuentran más cerca de un sujeto prototípico (1a), a diferencia de los sujetos auditivos involuntarios (1b), a los que justamente se les ha relacionado con la noción del sujeto experimentante, por su baja transitividad. Entre estos dos extremos de más o menos transitividad se encuentran los casos de la percepción auditiva voluntaria (escuchar) (1c) y la percepción visual involuntaria (ver) (1d).
a. Todos los días miramos el amanecer.
b. Juan oyó los disparos desde su casa.
c. Escuchamos la misma canción cada día.
d. Vemos a la chica cruzar la calle.

Sabiendo esto, el presente artículo se dedica a la posición del verbo sentir en este continuo. Como se desprende de la tabla 1, sentir suele caracterizarse como VdP involuntaria de la modalidad inferior del tacto (2), entrando en una oposición léxica con su homólogo de percepción activa, tocar (3):

Le hablé de [...] cómo me habían temblado las manos al sentir el roce de los labios de Nuria Monfort en la piel apenas unas horas atrás. [CREA: Ruíz Zafón, 2001]

(3) Alí viajará en helicóptero hasta una región del norte de Suecia, donde podrá tocar la nieve con sus manos. [CREA: Prensa, 2004]

Pese a su clasificación como verbo de percepción inferior, sentir se caracteriza con todo por una estructura sintáctica y semántica muy rica. En efecto, igual que los verbos de percepción dominantes, sentir admite una amplia gama de construcciones sintácticas. De esta manera, no solo admite OD nominales - con (4a) o sin determinante (4b) - sino que también se construye con complementos verbales: subordinadas completivas (5a), construcciones infinitivas (5b), de gerundio (5c) y oraciones relativas especificativas (6). Además, aparece en construcciones atributivas en las que el sujeto atribuye una propiedad particular al objeto percibido (7a-b).

(4) a. La tibieza de una mano le sujeta la frente. Y ella siente la mano de Tomasa. [CREA: Chacón D., 2002]

b. Francisco: No siento rencor, lo que sí siento es una gran indiferencia y, sobre todo, pena. [CREA: Prensa, 2004]

(5) a. Deberíamos recuperar, personal y colectivamente, el sentido de la muerte para mejorar nuestra frágil condición humana. [...] Para que sintieran que su poder es en sí mismo frágil y pedante; que sólo sirve si se arriesga al servicio de una causa grande. [CREA: Prensa, 1995] 
b. La sangre no deja de manar, el médico de Split era un inepto. La siente resbalar por su rostro, no es sangre lo que ve, ni sombra externa alguna lo que ocupa su retina, sino el catálogo de los hoteles de primera que frecuentó en el pasado. [CREA: Torres, M., 2004]

c. Podía sentir su sangre tibia empapándome la ropa. [España: Ruiz Zafón, C., 2001]

(6) Felipe la llamaba. Apresuró el paso. Para no sentir la congoja que le subía del estómago, comenzó a correr. [CREA: Chacón D., 2002]

(7) a. Y más aún, cuando al detenerse para coger el aire enrarecido que por el túnel circulaba escasamente sentía próximo el resuello del perseguidor, agrandado por los ecos interiores, como el de una alimaña. [CREA: Aguirre, F.J., 2000]

b. Personalmente tengo que decir que me siento muy satisfecho de haber trabajado con este hombre, discutido pero triunfador. [CREA: Del Rey del Val, 2002]

Esta riqueza sintáctica está relacionada con una intrincada opulencia semántica. Resulta claro que sentir no solo refiere a la percepción táctil (ejemplos 2, 4a, 5b, 5c) sino que también puede referir a otras modalidades de percepción física como la percepción auditiva (7a) e incluso la percepción física general (6). Por la presencia de la completiva en (5a), su significado se extiende hacia el campo de la cognición ('darse cuenta de'). En (4b), en cambio, hace referencia más bien a una sensación interna emotiva. La diferencia entre las frases (4a) y (4b) corresponde a la distinción entre la percepción interna (que refiere a una emoción experimentada por el sujeto desde dentro) y la percepción externa (causada por estímulos externos al cuerpo) (Viberg 2005: 129). ${ }^{3}$ Relacionados con estos contextos de percepción interna son los casos en los que el verbo denota una emoción más bien negativa de arrepentimiento. De hecho, sentir puede adoptar el significado específico de lamentar, tanto en su uso verbal pleno (8a) como en el uso discursivo de marcador de disculpa lo siento (8b):

a. Hay gente que se disculpa por sentarse aquí y hacerme preguntas. "siento molestarte". [CREA: Prensa, 2004]

b. Lo siento, señor, pero como estamos inmersos en esta operación me encontraba un poco despistada. [CREA: Giménez Bartlett, A., 2002]

La heterogeneidad de los ejemplos citados pone en tela de juicio la caracterización tradicional de sentir como verbo de percepción inferior del tacto. Aun así, subyacente a la serie de variaciones mencionadas, existe una base común: los distintos usos del verbo se caracterizan sintácticamente por el predominio del esquema transitivo.

El presente artículo se dedica precisamente al análisis de estas dos caras - una semántica otra sintáctica - de la transitividad de sentir en relación con su carácter multimodal y su polisemia. ¿En qué medida la transitividad sintáctica del verbo equivale a una transitividad semántica? A ese respecto, postulamos que el carácter multimodal del verbo está correlacionado con distintos grados de agentividad y control del sujeto, lo cual mina la validez de una rígida bipolaridad entre el sujeto agente vs. sujeto experimentante propuesta en la tradición lingüística. Por lo tanto, nuestro estudio se enmarca dentro de la corriente de trabajos lingüísticos que abogan a favor de la gradualidad de la transitividad aplicada al campo semántico de los VdP. Pero el análisis va más allá en el sentido de que no solo sitúa el verbo mismo en la escala de transitividad, sino que muestra que un solo verbo puede ocupar posiciones diferentes en función de su polisemia.

\footnotetext{
${ }^{3}$ Fernández Jaén (2006: 393) alude a la misma oposición mediante los términos endógena vs. exógena.
} 
El estudio se desarrolla en 3 fases. La Sección 2 proporciona más información sobre la constitución del corpus y el propio método de investigación. La Sección 3 se dedica a la descripción detallada de la polisemia del verbo, es decir se examina cuáles son los distintos significados adoptados por sentir y cómo se interrelacionan. A continuación, en la Sección 4 se estudiará, con base en datos empíricos, cómo se comporta el verbo sentir respecto a la definición de la transitividad prototípica.

\section{Metodología y datos generales}

Los datos de este estudio provienen de un muestreo al azar del paradigma entero del verbo, tomando en cuenta esencialmente dos criterios de selección, a saber el corte cronológico (1990-2006) y la variante diatópica (el español peninsular). A fin de obtener una muestra representativa, recopilamos tanto ejemplos orales como escritos. Más precisamente, para el corpus escrito, 400 casos han sido retenidos del Corpus de Referencia del Español Actual (CREA). Luego, este corpus ha sido complementado con el mismo número de datos orales extraídos de los corpus PRESEEA, COLAM y Val.es.co. ${ }^{4}$ Este corpus ha sido analizado y anotado manualmente por una amplia gama de propiedades que incluyen tanto características morfosintácticas como semánticas y pragmáticas. Más precisamente, distinguimos diversas variables correspondientes a cuatro niveles generales relativos al estudio de un verbo, a saber (1) las propiedades del verbo mismo (tiempo, modo, aspecto, persona y número gramatical), (2) la estructura argumental del verbo (forma: SN, pronombre, proposición; semántica: papel semántico, carácter +/- animado, etc.) (3) las características de otros complementos no argumentales (forma y papel semántico: lugar, meta, causa,...) y (4) fenómenos del discurso (ocurrencia del verbo en una predicación autónoma, colocaciones llamativas,...).

De manera general, el análisis semántico permite agrupar los significados en cinco núcleos semánticos, a saber, (1) la percepción física general, (2) la percepción física de modalidad específica, (3) la percepción emotiva, (4) la percepción cognitiva y (5) una categoría con casos ambiguos.

Como veremos más adelante (cf. Sección 3) no siempre resulta evidente distinguir claramente entre estos núcleos semánticos, puesto que los significados están estrechamente entrelazados entre sí y muchas veces no permiten un análisis discreto. Más bien vale reconocer la existencia de zonas transitorias y traslapes entre ellos y caracterizarlos en términos de continuidad. Por consiguiente, las decisiones de anotación semántica no siempre resultan unívocas. Obsérvese a título ilustrativo el ejemplo (9):

(9) Le besó la cara rozándole la piel y con una determinación que la sorprendió, la estrechó contra sí y posó sus labios en los de ella, primero suavemente, pero en seguida con fuerza, incluso hasta sentir que le hacía daño. [CREA: Casares Carlos, 1996]

En esta frase no se puede decir exactamente si se trata de una percepción física directa obtenida por el tacto (el contacto físico de los labios), o si al contrario, se trata de una percepción más bien indirecta que implica cierto razonamiento cognitivo: 'hasta darse cuenta

\footnotetext{
${ }^{4}$ Véase la bibliografía para las referencias completas del corpus.
} 
de que le hacía daño'. La presencia de este tipo de ejemplos justifica la inserción de una quinta categoría de 'casos ambiguos'.

Como la tarea de la clasificación de los significados es una parte esencial del presente estudio, los datos han sido analizados independientemente por dos lingüistas y con el objetivo de aumentar la fiabilidad del análisis, medidas de concordancia inter-observador han sido calculadas, a saber el coeficiente Kappa de Cohen, siguiendo a Glynn (2010). Como esta medida estadística tiene en cuenta el efecto del azar en las proporciones observadas, se considera generalmente como una medida más robusta que el mero cálculo del porcentaje de concordancia. Como regla general, si los observadores están en total acuerdo $\kappa=1$. Si no hay concordancia otra de la que se esperaría por azar, $\kappa=0$. En general, un coeficiente de $0.7 \mathrm{o}$ mayor indica una concordancia inter-observador prometedora. ${ }^{5}$ Para el estudio actual la concordancia entre observadores arrojó una concordancia que se acercó a $1(\kappa=0,89)$.

La tabla 2 visualiza las frecuencias de los significados del verbo según los cinco núcleos semánticos distinguidos:

\begin{tabular}{|l|l|l|}
\hline \multirow{2}{*}{} & \multicolumn{2}{|c|}{ SP } \\
\cline { 2 - 3 } & \multicolumn{1}{|c|}{$\%$} & \multicolumn{1}{c|}{$\%$} \\
\hline P física general & 53 & $6.6 \%$ \\
\hline P específica & 28 & $3.5 \%$ \\
\hline P cognitiva & 80 & $10 \%$ \\
\hline P emotiva & 583 & $72.9 \%$ \\
\hline Ambiguo & 56 & $7 \%$ \\
\hline Total & 800 & $100 \%$ \\
\hline
\end{tabular}

Tabla 2. Núcleos semánticos de sentir

Como ilustra este cuadro, el análisis semántico confirma claramente el carácter multimodal del verbo. En general sentir parece preferir los contextos emotivos (72.9\%), seguidos por los contextos de la percepción cognitiva (10\%) y la percepción física general (6.6\%). Sin embargo, como se especificará más adelante (Sección 3.2, 4.2.4), convendrá reconocer subclases y distinguir por ejemplo entre 'emociones' y 'reacciones emocionales'. Además, claro está que dentro de cada una de estas categorías semánticas generales, también hay diferencias semánticas más sutiles y que la frontera entre ellas no siempre es nítida, como ilustran los ejemplos ambiguos y los casos de desacuerdo inter-observador.

A continuación, se examinará primero más en detalle este carácter polisémico del verbo con base en las frecuencias globales. Intentamos responder a la pregunta cuáles son los distintos significados que puede adoptar este verbo y cómo se interrelacionan (Sección 3). Luego, tomando como base este perfil multimodal del verbo se verificará en qué medida los distintos significados están correlacionados con distintos grados de agentividad (Sección 4).

\footnotetext{
${ }^{5}$ Más específicamente, la potencia de concordancia se interpreta de la manera siguiente: $<0.2=$ débil; >0.2 $\leq$ 0,4 = bastante $>0.4 \leq 0.6=$ moderado $;>0.6 \leq 0.8=$ sustancial $;>0.8 \leq 1=$ muy bien .
} 


\section{La polisemia de sentir}

En el presente apartado, se examinará más en detalle este carácter polisémico del verbo con el objetivo de saber cuáles son los distintos significados que puede adoptar y cómo se interrelacionan.

\subsection{Sentir como verbo de percepción física}

El primer grupo semántico abarca los casos en los que sentir denota una percepción física realizada mediante los sentidos corporales. Como discutido en la Sección 1, sentir se ha relacionado habitualmente con el sentido del tacto (2), pero también puede referir a otras modalidades de percepción, como la auditiva (7a), la olfativa (10a) y la gustativa (10b).
a. Quiérete un poco. Panizo sintió la vaharada de la calle Narváez. - Qué fácil es decirlo, Marta. - Tú fíate de mí y no te obceques. [CREA: Longares, 2001]
b. El médico sacó la cajetilla de ducados y se la ofreció. Francisco encendió el cigarrillo y fumó en la soledad sin sentir el sabor del tabaco. [CREA: Castro, 1994]

Estos ejemplos confirman pues el carácter multimodal del verbo. Sin embargo, como se observa en la tabla 2, con base en las frecuencias, la referencia a una modalidad de percepción específica - aunque no imposible - resulta bastante excepcional (solo ocurre en el $3.5 \%$ del corpus total). De ello, podemos deducir que sentir no se puede definir como un verbo del tacto en sentido estricto.

En cambio, dentro del campo de la percepción física, el significado más frecuente (y por eso también más prototípico) resulta ser la percepción física general $(6.6 \%){ }^{6}$
El abrazo. Frotar las manos vigorosamente hasta calentarlas. Extender los brazos acostados con la palma hacia delante, los dedos extendidos, así sentiremos cómo la energía fluye por nuestros brazos e inspirando sentiremos cómo abarcamos el límite de nuestros lados, espirando los iremos recogiendo cruzando por delante del pecho sintiendo que abarcamos todo el perímetro hasta quedar con los brazos cruzados y las manos a los lados del cuerpo sobre los hombros o, si es posible, en la espalda (siempre sin forzar). [CREA: Pons Geis, 2001]

Este tipo de ejemplos ha llevado a autores como Iwasaki (2002: 33) a proponer una sexta modalidad de percepción, la 'propriocepción' o sensación interna, en la que sentir manifiesta "la conciencia del hablante de la existencia de su cuerpo sensible" (Fernández Jaén 2012: 417). El ejemplo (11) es muy ilustrativo a ese respecto. Este parámetro permite distinguir pues entre las sensaciones internas por un lado y las sensaciones que provienen del mundo externo por el otro, expresadas ambas posiblemente mediante el verbo sentir. Sin embargo, tanto las sensaciones originadas por agentes externos, como las producidas en el interior comparten el mismo eje o punto inicial: el cuerpo humano.

Esta importancia del cuerpo humano como punto de referencia para las orientaciones tanto hacia el exterior como hacia el interior se refleja al nivel sintáctico en la frecuente colocación

\footnotetext{
${ }^{6}$ Cabe precisar que la prototipicidad ha sido definida desde distintos puntos de vista y con base en varios criterios. Una manera de definir la prototipicidad es a través de la frecuencia: la idea subyacente es que significados más prototípicos simplemente ocurren más frecuentemente (cf. "From-Corpus-to-Cognition Principle” de Schmid 2000: 39).
} 
con un sintagma preposicional (SP) que precisa la localización exacta (generalmente una parte de cuerpo) de la sensación, sea interna (12a) sea externa (12b) al cuerpo humano:

(12) a. Soñé que Miquel me besaba en la frente y me tapaba con una estola. Al despertar sentí terribles punzadas de dolor en la cabeza que reconocí como el preludio de una resaca feroz. [CREA: Ruiz Zafón, 2001]

b. Siguió caminando y se quitó los zapatos cuando sintió bajo sus pies la arena blanda. [CREA: PérezReverte, 2002]

Tomando como punto de partida esta importancia del cuerpo humano, y reconociendo que la experiencia corporal siempre es el punto inicial para desarrollar significados más abstractos, no resulta sorprendente que haya extensiones hacia dominios más abstractos que establecen cierto contacto mental con un objeto mental o emocional. En otros términos sentir ilustra la consabida tendencia inherente a las unidades polisémicas de extender sus significados más concretos o físicos a ámbitos más abstractos (cf. Sweetser 1990).

El primer paso de lo concreto a lo abstracto se refleja claramente en unas proyecciones metafóricas que producen ejemplos a caballo entre ambos dominios. Así por ejemplo, es consabido que ciertas sensaciones físicas están estrechamente vinculadas a determinadas emociones, lo que favorece la aparición de contextos metafóricos.

\begin{abstract}
Sin afán de fabular o de dar a conocer su vivencia. Gracias a tanto charlatán y embaucador -pensaba en la soledad del automóvil-, a tanto vidente, a tanto vendedor de verdades espiritistas y esoteristas, a tanto abducido paranoico, a tanto profeta de saldo, los testigos como Iván debían sentir un nudo en la garganta. ¿Cómo contar su historia, estando envueltos del descrédito que hacia todo lo insólito han sembrado algunos miserables? [CREA: Jiménez, 2002]
\end{abstract}

En este ejemplo, se recurre a la descripción de una percepción física (el impedimento que se siente en la garganta y que estorba el tragar, hablar y algunas veces respirar) para en realidad referir a cierto estado emocional, a saber la aflicción que impide explicarse o hablar. La presencia de este tipo de ejemplos en la zona transitoria de lo físico y lo emotivo es bastante lógica, porque es consabido que la percepción física está relacionada metafóricamente a la percepción emotiva (cf. entre otros Sweetser 1990, Ibarretxe-Antuñano 1999, Kövecses 2008).

\title{
3.2 El núcleo emotivo
}

Los ejemplos emotivos con sentir son abundantes en el corpus (72.9\%). De acuerdo con Damasio (2003), entre otros, las emociones básicas - alegría, sorpresa, orgullo, tristeza, enfado, miedo y vergüenza - se dejan clasificar esencialmente en emociones positivas y negativas. Esto se manifiesta claramente en nuestros datos empíricos: sentir expresa con frecuencia este tipo de emociones tanto positivas (14a) como negativas (14b).

(14) a. Y Sofía sentía un orgullo intenso de compartir las horas con Patricia, sentía por primera vez que la vida no se le iba de las manos, sino que le venía al corazón directamente, densa y feliz. [CREA: Beccaria, 2001]

b. El individuo dio un paso al frente. Me retiré. No había sentido tanto miedo en la vida. [CREA: Ruiz Zafón, 2001] 
Sin embargo, los ejemplos emotivos con sentir no solo abarcan estas emociones básicas sino también emociones más elaboradas que requieren mayor esfuerzo cognitivo.

a. Todo está dentro de este libro que ya contiene la nostalgia futura que sentiré cuando recuerde el tiempo en el que fue escrito. [CREA: Lindo, 2001]

b. Aunque en el PSOE no está ni se le espera, tras su público apoyo al programa económico de Aznar, intelectualmente debe de seguir sintiendo la obligación de defenderlo. [CREA: Sotillos Palet, 2002]

Así por ejemplo, a diferencia de los ejemplos (14a) y (14b) donde se trata de una emoción básica que surge de manera más bien espontánea, en (15a) sentir nostalgia implica cierta conciencia del tiempo y exige la capacidad de recordar eventos del pasado e interpretarlos desde la perspectiva del presente. De hecho, son precisamente este tipo de ejemplos, donde el experimentante requiere de más capacidades cognitivas, que explican una parte sustancial de los casos de "desacuerdo inter-observador" a los que referimos en la Sección 2. En efecto, como ilustra el ejemplo (15b) sentir una obligación hace referencia a un estado emotivo actual del experimentante, pero también implica la posibilidad o la capacidad del experimentador de hacer proyecciones en el tiempo hacia eventos futuros. Este aumento del esfuerzo cognitivo se refleja también en las colocaciones. Por ejemplo, en la frase (15b) llama la atención la presencia del adverbio de modo intelectualmente que refiere claramente a la cognición, por lo que este tipo de ejemplos muestra los límites difusos de zona fronteriza entre la percepción emotiva y la percepción cognitiva.

\subsection{Sentir como verbo epistémico}

Los ejemplos anteriores se relacionan con los usos más claramente epistémicos del verbo.

\footnotetext{
a. Queridísima mía: Corre un rumor por la prisión, cada día más fuerte, y siento que cada día es más cierto que pronto estaremos juntos. [CREA: Chacón, 2002]

b. [...] lo confortable de aquellas reuniones, lo burgués, la sensación de seguridad y de reposo, invadían el corazón hasta anegarlo. Isabel de la Hoz sintió muy pronto que era obligatorio rebelarse contra aquel confortable reducto interior, como contra una tentación perversa: la tentación de no salir. [CREA: Pombo, 2004]

c. Analizando las cosas desde la distancia y fríamente, siento que en este país hay cosas que se pasan por alto. [CREA: Prensa, 2003]
}

Como se observa en (16a), en ciertos contextos sentir puede indicar una intuición equivalente a verbos como 'intuir' o 'presentir'. En (16b) se acerca al significado de 'darse cuenta de' o 'entender', mientras que el ejemplo (16c) vehicula más bien el significado de 'opinar'. El significado de percepción cognitiva se relaciona en la sintaxis con una completiva introducida por la conjunción que. Semánticamente hay un gran denominador común subyacente a todos estos ejemplos: el verbo no introduce certezas demostradas. Al contrario, introduce sospechas (16a) o admite usos epistémicos que expresan un descubrimiento involuntario basado en la experiencia (16b). Esto apunta a que en estos contextos epistémicos, sentir asume cierta función atenuativa bajando el nivel de agentividad con respecto a otros verbos cognitivos como 'saber' y 'pensar'. 
Relacionados asimismo con la percepción cognitiva son los casos en los que sentir denota cierta valoración mediante la cual se atribuye una cualidad a los objetos percibidos. Conceptualmente equivale a verbos como juzgar o considerar.

\footnotetext{
a. Un sadhu se sienta automáticamente a mi lado, como si me estuviera esperando. Se da por invitado, me mira con cierto descaro, sí, sonríe, tal vez se burla, tal vez me siente como su amigo espiritual, quizá le divierte mi actitud. [CREA: Calle, 2001]

b. Son hijos muy especiales, sus padres los sienten así. [CREA: Prensa, 2004]
}

La cualidad atribuida se expresa sintácticamente mediante un complemento predicativo introducido o no por como - que puede adoptar varias formas (sustantivo, adverbio, adjetivo). A diferencia de los usos epistémicos discutidos antes, en estos casos se trata de una percepción orientada hacia fenómenos externos en la que coinciden un estímulo externo con una sensación interna. Se trata pues de un cambio epistémico de subjetividad.

Finalmente, resulta interesante observar que esta importancia del 'yo consciente de su cuerpo sensible', presente de manera más o menos latente a través de todos estos núcleos semánticos del verbo sentir, también facilita la entrada del verbo en usos muy modalizados de alta subjetividad, bajo la forma de un marcador pragmático de disculpa ('lo siento').

\subsection{Resumen}

En suma, vimos cómo el verbo sentir codifica una amplia gama de significados: no solo puede expresar la percepción física general o específica, sino también se utiliza para la percepción emotiva, cognitiva y la valorización cognitiva. Sin embargo, como ya vimos, la asignación de una de estas clases al verbo no siempre resulta unívoca, lo que explica los casos de "desacuerdo inter-observador" y la frecuencia bastante elevada de casos ambiguos. La presencia de estos ejemplos sugiere un análisis en términos de continuidad semántica con claras ocurrencias del significado A convirtiéndose gradualmente en claras instancias del significado B y una amplia gama de ocurrencias situadas entre estos significados más prototípicos. En el apartado siguiente, se examinará cómo este carácter multimodal del verbo y su tendencia hacia mayor subjetividad está correlacionado con distintos grados de agentividad y control del experimentante.

\section{La transitividad del verbo: sentir en la jerarquía de control}

\subsection{Criterios de análisis}

Los diferentes dominios semánticos donde aparece sentir muestran un elemento en común, a saber la codificación de un sujeto-experimentante y un objeto-estímulo, es decir, sintácticamente sentir entra en el esquema transitivo S-V-OD. Sin embargo, ya sabemos que la relación icónica entre la transitividad sintáctica y la transitividad semántica - aunque predominante - no es perfecta. Como los VdP establecen una relación de contacto mental (en vez de material o físico) entre perceptor y percepto, sus participantes no coinciden con los roles prototípicos de agente y paciente, por lo cual han sido designados como verbos transitivos atípicos.

A fin de dar cuenta del grado distinto de agentividad, el sujeto de percepción ha sido identificado generalmente con el papel temático de experimentante (EXP). Sin embargo, la 
agentividad del participante sujeto no se define en términos discretos (Fillmore 1968, Cano Aguilar 1981, Demonte 1990), incluso dentro de este papel de EXP (Maldonado 1999). Más vale reconocer distintos grados de agentividad sujetiva y también varios EXPs que presentan distintos grados de agentividad. Con el fin de demostrar que la transitividad de los verbos mentales - y más particularmente también de los VdP - requiere una interpretación gradual, estos autores recurren a una serie de criterios semánticos y sintácticos que desvelan los diferentes tipos de EXPs.

En este estudio examinamos (1) en qué medida el carácter multimodal y la polisemia de sentir incide tanto en la capacidad del EXP para reflejar distintos grados de control como en el comportamiento del estímulo (EST), y (2) cómo la conjunción de ambos elementos se refleja en distintos grados de transitividad semántica. De acuerdo con estos objetivos aplicamos algunas de las pruebas referidas tanto al S-EXP como al O-EST del verbo sentir. Más específicamente, entre la amplia gama de criterios aducidos por lo que concierna al nivel de control del S-EXP, los que resultan relevantes para nuestro objeto de estudio son:

(a) el uso del imperativo

(b) el uso de adverbios de intencionalidad y adverbios de control

(c) la posibilidad de funcionar como complemento de verbos expresando una orden (convencer, obligar, persuadir), un acto volitivo (decidir) o de aparecer en perífrasis como dejar de + INF

(d) el uso de ciertas preposiciones

De manera similar, por lo que atañe al O-EST, dos propiedades esenciales han sido examinadas: [ \pm definitud] y [ \pm animacidad] (cf. Enghels 2013$)$.

4.2 El sujeto experimentante de sentir: grados de agentividad

\subsubsection{La jerarquía de Maldonado (1999)}

Tomamos como punto de partida la distinción que Maldonado (1999) establece entre los EXPs de los verbos mentales. A partir del Modelo de Roles Arquetípicos (Langacker 1991), plantea que hay dos patrones de extensión a partir de la constitución del EXP en contextos en que el verbo tiene dos argumentos: bien opera como el correlato abstracto del agente (con una transmisión de energía mental), bien el EXP se convierte en un paciente temático. Estos realineamientos están representados por las flechas en negrita en la figura siguiente:

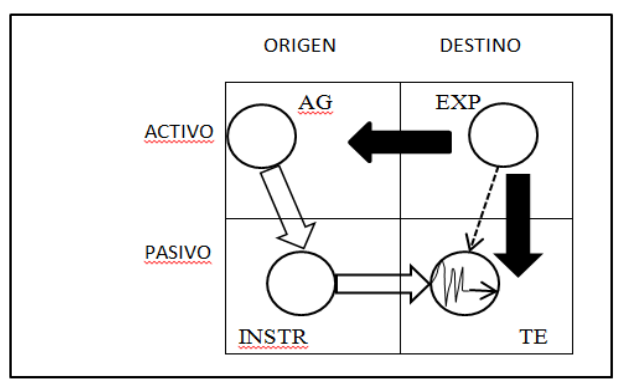

Figura 1. Ajuste de roles (Maldonado 1999)

De acuerdo con este esquema, los EXPS ACTIVOS son sujetos de corte agentivo ubicados en el dominio de origen y tienen capacidad de controlar sus acciones mientras que los EXPs PASIVOS son temas en el dominio de destino. Sufren un cambio de estado sobre el cual no tiene control. Este mayor o menor nivel de control del S-EXP depende de la clase verbal a la que pertenece. 
Así, Maldonado (1999) propone una jerarquía de control organizada en cuatro niveles de acuerdo con el grupo semántico verbal: ${ }^{7}$

\title{
PERCEPCIÓN > COGNICIÓN > EMOCIÓN > REACCIÓN EMOCIONAL
}

De acuerdo con esta escala, los verbos de percepción tienden a tomar un EXP activo; conforme se desciende en la jerarquía, aumenta la posibilidad de que ocurra un EXP pasivo. Como se ha mostrado en la Sección 3, por su carácter polisémico el verbo sentir aparece tanto en contextos perceptivos como cognitivos y emotivos. Por ello, conviene examinar en qué medida este carácter polisémico del verbo incide también en su nivel de control, o más precisamente, en su capacidad de reflejar distintos grados de control.

A fin de dar cuenta de las diferencias respecto del nivel de actividad del EXP, Maldonado plantea una correlación entre la direccionalidad y el nivel de control. El control opera precisamente en relación directa con el flujo natural de transmisión de energía cuya orientación básica es ORIGEN > DESTINO, mientras que si el flujo es opuesto DESTINO > ORIGEN, éste se relaciona con un menor control. Aunque con el verbo sentir la acción suele proyectarse del dominio de destino al de origen [DESTINO > ORIGEN] (ejemplo 18a), o a veces incluso carece de orientación (18b), no se descartan los usos con la dirección ORIGEN > DESTINO (18c), relacionados con mayor control y agentividad:

\begin{abstract}
a. Una terrible certeza me golpeó de súbito. Aquel hombre era el extraño que abordaba a Clara por la calle, de incógnito. Era real. Clara no había mentido. El individuo dio un paso al frente. Me retiré. No había sentido tanto miedo en la vida. [CREA: Ruiz Zafón, 2001]

b. Mi novio me invito a acompañarlo a su examen profesional, pero ojo él vive en una ciudad bastante lejos y me pago todos los gastos, vaya que le salió caro, ya que se necesitan tomar un avión, y dos autobuses para llegar a ese lugar mínimo 1 día. Me ama tanto que siento que no lo merezco. [CREA: Prensa, 2003]

c. Cuando se meta en cama, olvídese de todo y piense en algo que le guste: imagínese dándose un chapuzón y sienta el agua, la suavidad de la toalla al secarse, la caricia del sol, o vuele por encima de un paisaje idílico y perciba el viento en el rostro, la sensación de libertad, la ingravidez... [CREA: Estivill E., 1997]
\end{abstract}

Estos ejemplos ilustran las diferentes orientaciones del flujo de energía que sentir puede presentar. El ejemplo (18a) que corresponde al dominio emocional presenta una orientación contraria al flujo natural, por lo que su EXP no presenta un alto grado de control. Otros contextos de sentir, como el del dominio cognitivo (18b), pueden carecer de una orientación de energía mental para que el EXP la regule. A diferencia del resto de ejemplos, el contexto físico de (18c), cuya transmisión de energía es ORIGEN > DESTINO, muestra claramente que el EXP posee un mayor nivel de control, es decir, utiliza su propia energía para realizar el proceso de percepción, siendo la causa directa del proceso (Enghels 2013).

\footnotetext{
${ }^{7}$ En lugar de la división de los verbos mentales en tres clases básicas (Croft 1986), Maldonado (1999) incluye el grupo de 'reacción emocional' que tienen un EXP pasivo y un sujeto inductor del cambio emocional que puede ser activo (agentivo) o inactivo (instrumental).
} 


\subsubsection{El sujeto-perceptor}

El grado más agentivo de estos casos de la percepción física se valora mediante varias pruebas formales. Así, está claro que los contextos más agentivos permitirán el uso del imperativo, como se observa en la forma verbal sienta (18c), porque solo los verbos con alto nivel de control pueden codificar órdenes. Otra prueba es que admiten el acompañamiento de adverbios de intencionalidad del tipo intensamente o deliberadamente que controlan la intensidad con la que se realiza una acción. Este tipo de adverbio se añade por ejemplo fácilmente al caso siguiente:

$$
\text { Cuando dejé Vietnam tu madre estaba embarazada de nueve meses y yo solía poner mi mano en su }
$$
barriga para sentir [deliberadamente] tus patadas. [CREA: Prensa, 2003]

Asimismo, cuando codifica la percepción física, sentir tiene la posibilidad de ocurrir en perífrasis que indican volición (20a) o de funcionar como el complemento de verbos rectores de intención (20b) y de obligación (20c):
a. Comenzaremos por abrazarnos al árbol durante unos minutos, respirando profundamente y haciendo una especie de relajación sensitiva, tratando de sentir la propia energía del árbol. [CREA: Iborra Montells, 2001]
b. Pues allí está el Papa, como una encendida estrella a la que todo el mundo quiere tocar y sentir. [CREA: Prensa, 2003]
c. Cuando hablamos de cualquier actividad física, también nos referimos a la respiración, ya que no hay vida ni movimiento sin respiración. Automáticamente, al realizar ejercicio, debemos sentir el aire, combinar una respiración lenta o mejor dicho adecuada al movimiento. [CREA: Pons Geis, 2001]

De estas pruebas se deduce pues que el verbo sentir, cuando codifica la percepción física, puede aparecer en contextos de mayor control y agentividad sujetiva.

Sin embargo, como indica Maldonado (1999), conviene distinguir bien entre dos tipos de control: el que se establece sobre el objeto y el que el propio sujeto ejerce sobre su actividad mental. Este último tipo se refleja de manera más clara en los usos epistémicos del verbo sentir.

\subsubsection{El sujeto epistémico}

Como ya aclaramos en la Sección 3.3, muchas veces sentir codifica un tipo de conocimiento que no requiere mucho compromiso epistémico por parte del hablante. De la misma manera, como se observa en (21), aunque el participante está consciente de su actividad mental, no tiene la capacidad de dirigirla.

\footnotetext{
Analizando las cosas desde la distancia y fríamente, siento que en este país hay cosas que se pasan por alto. [CREA: Prensa, 2003]
}

Como se observa en este ejemplo, el complemento introduce un contenido proposicional que, con base en una serie de razonamientos previos, ha pasado a formar parte integrante del conocimiento del sujeto. En otros términos, el objeto mental está situado dentro del dominio del EXP, lo que refleja a su vez un nivel más bajo de diferenciación entre el sujeto y el objeto mental. Este menor control sobre la actividad mental hace que sentir en estos casos epistémicos asuma cierta función atenuativa bajando el nivel de agentividad con respecto a 
otros verbos cognitivos más agentivos como 'pensar' y 'saber'. Por este menor grado de control, estos usos producen resultados cuestionables frente a los contextos que ponen a prueba el nivel de control del S.

$$
\text { *Sienta / Siento deliberadamente que en este país hay cosas que [...]. }
$$

Sin embargo, conviene recordar que un subtipo de la percepción cognitiva es la percepción valorativa (cf. Sección 3.3). A diferencia de los usos epistémicos antedichos, la percepción valorativa es el resultado de un proceso activo de evaluación modal que requiere un acto consciente de reflexión (Hanegreefs 2008: 140).

(23) En efecto, creemos que la izquierda abertzale siente a la Organización debilitada, sin capacidad de influir decisivamente en su quehacer armado. [CREA: Prensa, 2004]

Además, este mayor nivel de control se refleja en la mayor diferenciación entre el sujeto y el objeto por atribuir una cualidad a cierto objeto externo percibido, al que se vincula cierta valoración personal.

\subsubsection{El sujeto emotivo}

En cuanto al dominio de la percepción emotiva, los datos empíricos de sentir justifican la distinción entre verbos de emoción y verbos de reacción emocional, los primeros representando un nivel de control intermedio, mientras que los verbos de reacción emocional tienen un EXP de control nulo sobre el desarrollo de la acción. Esta diferencia se verifica mediante varias pruebas. Así, por ejemplo, Bogard (1995) sostiene que la distinción de dos tipos de verbos de emoción se muestra sensible al empleo de preposiciones. Más concretamente, es de esperar que los verbos con un EXP más agentivo se contruyan con preposiciones cuya valencia refleja una organización ORIGEN > DESTINO. En efecto, sentir opera frecuentemente con preposiciones explicitando la meta del objeto emocional.

Los marroquíes sienten una fuerte atracción hacia el lujo material, y por extensión hacia los hoteles y casas que lo tienen, incluso aunque no sea mucho para el paladar europeo. [CREA: Silva, 2001]

Además, aunque en ambos tipos de emociones el sujeto no tiene realmente control sobre la acción misma, cuando sentir codifica una emoción, puede haber distintos grados de intensidad (cf. por ejemplo la presencia de adjetivos como profundo y adverbios como intensamente, profundamente, etc.). En los casos de reacción emocional, en cambio, incluso la intensificación tiene restricciones. En efecto, como afirma Maldonado (1999), los intensificadores con que opera la reacción emocional son consistentemente de bajo nivel de elaboración (por ejemplo mucho y no con todo el corazón o mesuradamente). En nuestro corpus, este tipo de adverbios de poca elaboración surgen con mucha frecuencia en los contextos de reacción emocional equivalente a 'lamentar'.

(25) No llores, mamá -Sara tiró el barreño al suelo y fue hacia ella, ahogándose en sollozos más violentos-. Yo... lo siento mucho... [CREA: Grandes, 2002] 
Está claro pues que como verbo de percepción, cognición y emoción, sentir tiene distintos grados de control. En cambio, los verbos de reacción emocional contrastan en nivel de control, porque no hay control alguno del EXP. En estos casos, un estímulo inactivo induce un cambio mental en un EXP PASIVO. Los ejemplos con el verbo sentir como reacción emocional abundan en el corpus:

A pesar de la alegría que sentían, aquel día, casi en el mismo instante en que acabó la película, empezaron a discutir por una insignificancia. [CREA: Casares, 1996]

Además, llama la atención cómo este nivel nulo de control está asociado con menor diferenciación entre el sujeto y el objeto: la diferenciación entre los participantes deja de ser nítida, puesto que es difícil establecer una separación clara respecto de nuestras emociones. A este factor de diferenciación nula entre sujeto cognosciente y objeto mental, se suma el factor de la direccionalidad de la experiencia emocional, en que el EXP se ubica en el dominio de destino (cf. Figura 1) y bajo la influencia de un flujo de energía proveniente del campo de origen, en otros términos presentando la orientación DESTINO > ORIGEN.

Es precisamente el nivel de actividad bajo del EXP en el dominio emotivo que facilita la entrada del verbo en contextos de voz media.

Teníamos de maestro al reverendo Kemrap Norbula, que en aquella época era el doctor más experto del país. Me siento muy feliz de haber sido su alumno. [CREA: Calle, 2001]

La combinación de la forma pronominal se con verbos de reacción emocional tiene como resultado que el EXP sufra un cambio emocional que no es capaz de controlar volitivamente. Por esta falta de los rasgos [+volitivo] y [+control], se ha reconocido en el clítico se un signo intransitivizador: elimina uno de los argumentos del lexema verbal que por consiguiente refiere indistintamente a un solo participante. Sin embargo, relacionado con el valor de este se, Maldonado (1999: 106) precisa que "si la distancia entre sujeto y objeto se reduce de manera tal que la diferenciación entre participantes deja de ser nítida, el clítico se produce construcciones medias cuyo EXP medio tiene participación alta y control nulo".

En otros términos, aunque el control sigue siendo nulo - reflejando por consiguiente un nivel de transitividad bajo - la construcción media del verbo sí aumenta la participación sujetiva del EXP. Esto es, se activa y aumenta la participación en el acto emotivo, pero esta mayor participación no implica mayor control. Una prueba alegada por Maldonado (1999: 98) para evidenciar este nivel de participación sujetiva se observa en el uso del imperativo directo. Solo con la forma se los verbos de reacción emocional pueden ocurrir en forma imperativa: * ;Siente alegría / gusto! Esta es tu casa vs. ¡Siéntete alegre / a gusto! Esta es tu casa. De acuerdo con Fernández Jaén (2012: 444) y como se observa en (27), los predicativos del sujeto resultan de una subjetividad muy elevada: al sentirse "feliz" o "orgulloso" el EXP evalúa su estado interno para llegar a una conclusión altamente modalizada: precisamente el hecho de que con sentirse la evaluación sea interna implica que es un proceso fuertemente subjetivo (cf. Lakoff 1996). ${ }^{8}$

\footnotetext{
${ }^{8}$ Lakoff (1996) recurre a la metáfora de LA PERSONA DIVIDIVA para explicar la capacidad del ser humano para conceptualizarse a sí mismo de modo dual. Según esta metáfora, las personas conceptualizan la reflexividad
} 


\subsubsection{Resumen}

Queda expuesto que el verbo sentir recorre el camino entero de la escala de control y transitividad inherente a los verbos mentales. Si bien el nivel de control está determinado por las características semánticas de cada verbo mental, aquí hemos demostrado que incluso el sujeto EXP de un mismo verbo presenta diferencias en cuanto a su nivel de control y agentividad. Por lo tanto, sentir no codifica un solo tipo de EXP, sino que presenta diferentes EXPs con comportamientos semánticos y sintácticos diferenciados: a partir de su uso como verbo de percepción física con control alto y EXP activo, se comprueba cómo el nivel de control baja conforme desciende en la escala, pasando por usos cognitivos y emocionales hasta convertirse en un EXP pasivo con control nulo en el dominio de la reacción emocional. Además, comprobamos cómo - paralelamente a este descenso de control sujetivo - la clara diferenciación entre el sujeto y el objeto se desvanece resultando en un verbo de reacción emocional donde desaparece por completo la posibilidad de distinguir entre ambos participantes. Finalmente, dada la relación inversamente proporcional entre la agentividad y la subjetividad podríamos pensar que es precisamente este bajo nivel de agentividad en los escalones inferiores de la escala lo que condiciona la posibilidad de aparecer en contextos de mayor subjetividad.

Aparte de esta caracterización del sujeto-experimentante, también es necesario examinar más en detalle el comportamiento aislado del objeto para verificar si este también refleja distintos grados transitividad.

\subsection{Características del objeto-estímulo}

El comportamiento del objeto también proporciona información acerca de la transitividad de sentir. De hecho, ya hemos visto cómo la diferenciación entre el sujeto y el objeto da indicios de un mayor control y, por ende, mayor transitividad. Ahora bien, focalizando en las características del objeto, ha sido argumentado que el OD estímulo es un paciente no prototípico porque no está afectado por el proceso de percepción (Langacker 1991: 13; Van Valin y Lapolla 1997: 145). Sin embargo, podríamos pensar que - paralelamente a la escala de agentividad del sujeto - también el objeto presenta distintos grados de prototipicidad. A ese respecto, y siguiendo a Enghels (2013), analizaremos dos rasgos semánticos específicos del objeto, a saber el grado de definitud (4.3.1) y la animacidad (4.3.2).

\subsubsection{La definitud del objeto-estímulo}

Primero, Kirsner y Thompson (1976: 227) argumentan que los EXPs voluntarios y más agentivos normalmente deben seleccionar ODs definidos e individualizados. De acuerdo con Enghels (2007) consideramos entre los estímulos definidos [+ DEF]: nombres propios, y SSNN introducidos por el artículo definido, un demostrativo o un adjetivo posesivo. Los

\footnotetext{
a partir de dos entidades: el SUJETO y UNO MISMO. El SUJETO es el locus de la experiencia subjetiva: la conciencia, la percepción, la racionalidad. Por el contrario, UNO MISMO incluye las características físicas del cuerpo. La relación entre ambos conceptos es espacial: UNO MISMO es el contenedor donde está el SUJETO. Si el SUJETO está dentro de UNO MISMO es más subjetivo, mientras que cuando el SUJETO está fuera de UNO MISMO es más objetivo. Como explica Lakoff, uno normalmente está dentro de sí mismo y salir afuera implica más esfuerzo y más control que quedar dentro. Por consiguiente, uno normalmente es subjetivo, y ser objetivo implica más esfuerzo y más control que ser subjetivo. Claro está que es la semántica original del verbo sentir, por su vínculo estrecho con la corporeidad y el 'yo consciente de su cuerpo sensible', la que permite la entrada del verbo en estos contextos de alta subjetividad.
} 
estímulos indefinidos [-DEF] son SSNN introducidos por un artículo indefinido, un adjetivo numérico o un cuantificador indefinido, o simplemente con nombres escuetos. Un análisis de los casos de sentir con un OD bajo forma de SN arroja los resultados siguientes:

\begin{tabular}{|l|c|c|c|c|c|c|}
\hline \multirow{2}{*}{} & \multicolumn{2}{|c|}{+ DEF } & \multicolumn{2}{c|}{ - DEF } & \multicolumn{2}{c|}{ Total } \\
\cline { 2 - 7 } & $\#$ & $\%$ & $\#$ & $\%$ & $\#$ & $\%$ \\
\hline P fís esp & 14 & $87,5 \%$ & 2 & $12,5 \%$ & 16 & $100 \%$ \\
\hline P fís gen & 12 & $50,0 \%$ & 12 & $50,0 \%$ & 24 & $100 \%$ \\
\hline P cogn & 4 & $100,0 \%$ & 0 & $0,0 \%$ & 4 & $100 \%$ \\
\hline P emo & 27 & $29,6 \%$ & 64 & $70,4 \%$ & 91 & $100 \%$ \\
\hline
\end{tabular}

Tabla 3. Definitud de estímulos con sentir + FN

De manera general (y excepción hecha de la percepción cognitiva, dado el número muy limitado de casos representativos), este cuadro muestra que el porcentaje de los objetos definidos es más alto en los casos de la percepción física y disminuye fuertemente con la percepción emotiva. En otros términos, paralelamente a su posición más alta en la escala de agentividad del sujeto, la percepción física se combina más frecuentemente con objetos más prototípicos, por lo que ésta representa el grado más alto de transitividad, mientras que la percepción emotiva se construye con objetos menos prototípicos. En el campo de la percepción emotiva, conviene destacar la alta frecuencia de ODs escuetos.

¿Es que vienes de otro lugar, Jorge, qué te pasa? ¿Por qué me da la sensación de que no sientes temor alguno, de que no tienes un ápice de miedo? [CREA: Beccaria, 2001]

Este grupo reúne los casos como sentir miedo, sentir vergüenza, sentir alegría, sentir sorpresa, etc. donde sentir se comporta como un verbo ligero en un predicado complejo $\mathrm{V}+$ OD abstracto, equivalente a verbos simples como temer, avergonzar(se), alegrar(se), sorprender(se). Está claro que en estos contextos el verbo se caracteriza por un menor grado de transitividad.

\subsubsection{El carácter [+/- animado] del objeto-estímulo}

La misma diferencia de transitividad se observa en el carácter [ \pm animado] del objeto. En general, se acepta que el paciente prototípico es una entidad inanimada. ${ }^{9}$ El análisis del corpus revela que sentir admite tanto objetos inanimados como animados: $:^{10}$

\begin{tabular}{|l|c|c|c|c|c|c|}
\hline \multirow{2}{*}{} & \multicolumn{2}{|c|}{+ ANIM } & \multicolumn{2}{c|}{ - ANIM } & \multicolumn{2}{c|}{ Total } \\
\cline { 2 - 7 } & $\#$ & $\%$ & $\#$ & \% & $\#$ & $\%$ \\
\hline P fís esp & 17 & $50,0 \%$ & 17 & $50,0 \%$ & 34 & 100 \\
\hline P fís gen & 1 & $3,6 \%$ & 27 & $96,4 \%$ & 28 & 100 \\
\hline P cogn & 26 & $70,3 \%$ & 11 & $29,7 \%$ & 37 & 100 \\
\hline P emo & 354 & $74,1 \%$ & 124 & $25,9 \%$ & 478 & 100 \\
\hline
\end{tabular}

Tabla 4. Animacidad de estímulo con sentir

En general, observamos una preferencia por objetos inanimados con la percepción física que disminuye considerablemente en la percepción cognitiva y emotiva. En otros términos, otra

\footnotetext{
${ }^{9}$ Para argumentos a favor y en contra de esta visión, véase Enghels (2013: 38).

${ }^{10}$ Para este análisis del rasgo [ \pm animado] solo tomamos en cuenta los casos en los que el objeto de sentir refiere a una entidad, representada por nombres propios, SNNN y pronombres excluyendo pues los casos que refieren a un evento bajo la forma de una proposición.
} 
vez está claro que la percepción física se construye de preferencia con objetos prototípicos, lo que incide en un grado más alto de transitividad.

(29) a. Es decir, cuando ella estaba, estaba y estaba a tope. Tan a tope que cuando no estaba seguíamos teniéndola a tope. Ahora mismo no está y yo todavía la siento, siento su olor ¿no? muchas veces. [CREA: oral, 1995]

b. Piensa en las lagartijas que ahora duermen bajo las piedras calientes y a salvo de navajazos, evoca los ojos trabados de Paulino sumidos en su muda paciencia [...] , y siente el frío hocico de Chispa, que prolonga su existencia pegado a sus tobillos lastimados, husmeando aromas de arañazos y tintura de yodo. [CREA: Marsé, 2000]

c. Estando ahora tan lejos era cuando la sentía más próxima a mí, en la distancia y en las cartas, en mi ignorancia casi absoluta sobre la vida que llevaba. [Muñoz Molina, Sefarad, 2001]

d. Me siento satisfecho de poder formar parte de un importante grupo de investigación, Conservation International, en el que hay gente que ha puesto su cerebro o su dinero al servicio de un problema tan complejo y decisivo. [CREA: Prensa, 2003]

En los ejemplos de percepción física, aunque pueden aparecer entidades humanas (29a), esto resulta muy raro; generalmente prefiere entidades inanimadas como (29b). En la percepción cognitiva, aunque pueden aparecer objetos inanimados, suelen ser preferentemente entidades humanas. Los casos de la cognición valorativa son muy ilustrativos al respecto, donde se atribuye una cualidad a una persona de quien se dicta un juicio (29c). Sin embargo, la alta frecuencia de objetos animados en los dominios cognitivos y emotivos probablemente se explica por los casos de correferencia con el experimentante, respecto del cual se describe un estado emocional (29d). Respecto de este último ejemplo, es interesante observar que el sujeto y el objeto son correferenciales y el objeto funciona como una especie de receptor del estado anímico, ya no como un estímulo. De hecho el estímulo coaparece con el receptor codificado como una oración subordinada causal (de poder formar parte de un importante grupo de investigación...). ${ }^{11}$

Así, está claro que los objetos de sentir pueden denotar entidades humanas o no humanas. Sin embargo, la percepción física es la que suele codificar menos objetos humanos, mientras que la emotiva es la que muestra esta preferencia. Esta inestabilidad y en algunas ocasiones preferencia por las entidades humanas, nos habla de la lejanía con el paciente prototípico. Además, observamos cómo la semántica del objeto de sentir varía entre la de un locus receptor de valoraciones y estados emotivos y la de causa de una reacción emocional en el experimentante.

\subsection{Caso particular: sentir como marcador pragmático-discursivo}

Antes de pasar a las conclusiones de este estudio, conviene detenerse un momento en un uso muy particular del verbo sentir en español, y las consecuencias que tiene para el análisis de su grado de transitividad. Efectivamente, sentir también se puede utilizar para la expresión del dolor del otro o de un dolor compartido. En este sentido, el verbo equivale a lamentar, tanto en su uso verbal pleno (30a) como en la expresión fija lo siento (30b):

\footnotetext{
${ }^{11}$ En efecto, el objeto no necesariamente coincide con el papel de estímulo. Prueba de eso es que existen oraciones en las que conviven el objeto-locativo receptor y el estímulo codificado por una oración subordinada: [Me] siento cansada [por tanto ejercicio].
} 
a. Yo... No sé lo que me pasa. Me siento mal, muy mal, peor que nunca... Pero te quiero, Tam, y siento mucho haberme puesto así. [CREA: Grandes, 2002]

b. Yo necesitaba marcar goles y el penalti era una buena oportunidad de hacerlo, pero sé que él también necesitaba marcar, porque llevaba algún tiempo sin jugar por culpa de las lesiones. La pena es que no pudiera hacer gol y lo siento de verdad por él". [CREA: Prensa, 1999]

Algunos ejemplos incluso indican que su significado actual no se limita a la expresión de arrepentimiento equivalente a lamentar, sino que en determinadas circunstancias incluso parece extenderse hacia el campo de las llamadas 'emociones sociales' (Damasio 2003: 4345):

\begin{abstract}
Por primera vez perdió la compostura y elevó la voz. - No creo que los problemas médicos de los ciudadanos sean asunto de la policía.- Lo siento, Rosa, pero en este caso sí lo son. ¿Cuánto tiempo permaneció en la consulta del ginecólogo? - No sé, no me acuerdo. ¿Adónde quiere ir a parar? [CREA: Giménez Bartlett, 2002]
\end{abstract}

Está claro que este uso de lo siento ya se ha desemantizado por completo puesto que ya no implica arrepentimiento en sentido estricto sino que 'lo siento' en estos casos es una predicación autónoma que funciona como marcador pragmático intersubjetivo. ${ }^{12}$

La desemantización del verbo se refleja también en la sintaxis y más precisamente en una debilitación de la transitividad del verbo. Así, llama la atención que siempre aparece en una estructura argumental debilitada, con un OD no léxico, inestable y además, suele ocurrir en la primera persona del singular, es decir, la persona más subjetiva y más alta en la jerarquía de empatía. En otros términos, 'lo siento' hoy día se ha convertido en una especie de fórmula retórica, caso fronterizo de la transitividad, por lo cual ya se puede considerar como una construcción, funcionando como un marcador pragmático. Una prueba contundente a favor de la idea de que en este uso pragmático 'lo' se vacía más, hasta el punto de que ya no se considera OD del verbo, se encuentra en el ejemplo siguiente:

\begin{abstract}
¿Piensas que el caso Urquijo está ya cerrado? Bueno, lo siento decirte una cosa, Nieves. Que no quieres hablar pero prefiero no seguir hablando del tema, porque creo que, bueno, que lo que ha pasado ha pasado, y que no voy a seguir durante uno uno años hablando de las mismas cosas [CREA: oral, 1991].
\end{abstract}

En este ejemplo, llama la atención la presencia de dos ODs, tanto el pronombre 'lo' como el infinitivo 'decirte', por lo cual se deduce que en esta fórmula 'lo siento', 'lo' constituye una unidad léxica con el $\mathrm{V}$ y ya pierde referencialidad y estatus argumental.

\title{
5. Conclusión
}

Este estudio sobre la semántica del verbo sentir lleva a distintas conclusiones en varios niveles de análisis. En primer lugar, dentro del panorama más amplio de los verbos de percepción, resulta que sentir - tal como los verbos dominantes de la visión y del oído también presenta un perfil semántico muy rico - que se extiende desde significados de

\footnotetext{
${ }^{12}$ Cf. por ejemplo Aijmer \& Simon-Vandenbergen (2011) acerca de la noción de 'pragmatic marker'.
} 
percepción física hacia significados más abstractos epistémicos y emocionales e incluso contextos más pragmáticos - relacionado a una amplia gama de construcciones sintácticas.

Tomando como punto de partida este carácter multimodal del verbo y su preferencia por el esquema transitivo como denominador común subyacente a todos esos usos, examinamos a continuación en qué medida esta transitividad sintáctica del verbo refleja también una transitividad semántica, prestando atención tanto al grado de agentividad y control del sujetoEXP como a las características prototípicas del objeto-EST. Comprobamos cómo el verbo sentir recorre el camino entero de la escala de control y transitividad inherente a los verbos mentales: no codifica un solo tipo de EXP, sino que presenta diferentes EXPs con comportamientos semánticos y sintácticos diferenciados: a partir de su uso como verbo de percepción física con control alto y EXP activo, vimos cómo el nivel de control baja conforme desciende en la escala, pasando por usos cognitivos y emocionales hasta convertirse en un EXP pasivo con control nulo en el dominio de la reacción emocional. Además, vimos los objetosestímulos también se vuelven menos prototípicos según la misma escala. En un nivel más general, el caso de sentir ilustra que la distinción rígida agente/experimentante propuesta en la tradición lingüística no es sostenible y que más bien vale reconocer el carácter gradual de la agentividad con los verbos de percepción. En este sentido, nuestro análisis valida los resultados de algunos estudios previos sobre el carácter gradual de la transitividad en este grupo semántico, pero además demuestra que incluso dentro de un mismo verbo polisémico conviene distinguir distintos grados de transitividad, según la escala siguiente:

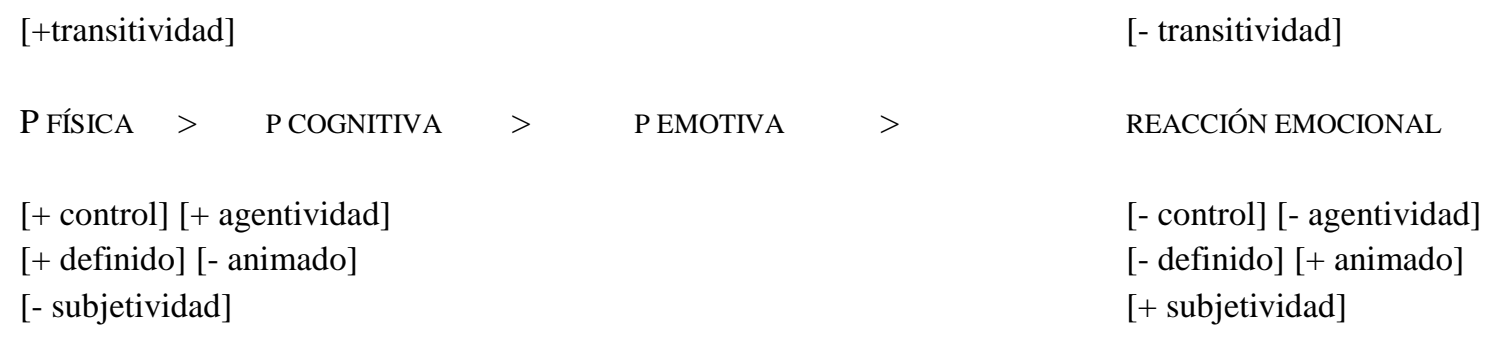

\section{Referencias}

Corpus:

Corpus oral de lenguaje adolescente de Madrid, http://www.colam.org [COLAM].

Preseea, Proyecto para el estudio sociolingüístico del español de España y América, http://preseea.linguas.net/Corpus.aspx [PRESEEA].

Real Academia Española, Corpus de Referencia del Español Actual, http://www.rae.es/ [CREA].

Val.Es.Co. Valencia Español Coloquial. Corpus de español coloquial, http://www.uv.es/corpusvalesco/index.html [Val.es.co]

Obras citadas:

Aijmer, Karin/Simon-Vandenbergen, Anne-Marie, Pragmatic markers, in: Zienkowski, Jan/ Östman, Jan-Ola/Verschueren Jef (edd.), Discursive Pragmatics, 2011, 223-247. 
Bogard Sierra, Sergio Eduardo, El argumento no experimentante de los verbos psicológicos: ¿ un papel temático o dos?, Nueva Revista de Filología Hispánica, 43/2 (1995), 441454.

Cano Aguilar, Rafael. Estructuras Sintácticas Transitivas en el Español Actual, Madrid, Gredos, 1981.

Croft, William, Categories and Relations in Syntax. The Clause-Level Organization of Information, Palo Alto, Universidad de Stanford, 1986.

Damasio, Antonio, Looking for Spinoza. Joy, Sorrow, and the Feeling Brain, Orlando, Harcourt, 2003.

Demonte Violeta, Transitividad, intransitividad y papeles temáticos, in: Garza Cuarón, Beatriz/Demonte, Violeta (edd.), Estudios de Lingüística de España y de México, México, El Colegio de México-UNAM, 1990, 115-150.

Dowty, David, Thematic protoroles and argument selection. Language 67/3 (1991), 547-619.

Enghels, Renata, Les modalités de perception visuelle et auditive: différences conceptuelles et répercussions sémantico-syntaxiques en espagnol et en français, Tübingen, Niemeyer, 2007.

Enghels, Renata, Transitivity of Spanish perception verbs: a gradual category? Borealis: An International Journal of Hispanic Linguistics, 2/1 (2013), 35-56.

Fernández Jaén, Jorge, Verbos de percepción sensorial en español: una clasificación cognitiva, Interlingüística 16 (2006), 391-405.

Fernández Jaén, Jorge, Semántica cognitiva diacrónica de los verbos de percepción física del español, Alicante, Universidad de Alicante, 2012.

Fillmore, Charles J., The Case for Case, in: Bach, Emmon/Robert T. Harms (edd.), Universals in linguistic theory, New York, Holt, Rinehart \& Winston, 1968, 1-90.

García-Miguel, José M., Transitividad y complementación preposicional en español, Santiago de Compostela, Universidad de Santiago, 1995.

García-Miguel, José M., Aproximación empírica a la interacción de verbos y esquemas construccionales, ejemplificada con los verbos de percepción, Estudios de Lingüística 19 (1995), 169-191.

Geisler, Hans, Das Verhältnis von semantischer und syntaktischer Transitivität im Französischen, Rjb 39 (1989), 22-35.

Glynn, Dylan, Testing the Hypothesis. Objectivity and Verification in Usage-Based Cognitive Semantics, in: Glynn, Dylan/Fischer Kerstin (edd.), Quantitative Cognitive Semantics. Corpus-driven approaches, Berlin, Mouton de Gruyter, 2010, 239-270.

Gruber, Jeffrey S., Look and see, Language 43/4 (1967), 937-947.

Hanegreefs, Hilde, Los verbos de percepción visual. Un análisis de corpus en un marco cognitivo, Leuven, KULeuven, 2008.

Hopper, Paul J./Thompson, Sandra A., Transitivity in grammar and discourse, Language 56/2 (1980), 251-299.

Ibarretxe-Antuñano, Iraide B., Polysemy and metaphor in perception verbs: a cross-linguistic study, University of Edinburgh, 1999.

Iwasaki, Shoichi, Proprioceptive-state expressions in Thai, Studies in Language, 26/1 (2002), 33-66. 
Kirsner, Robert S./Thompson, Sandra A., The role of pragmatic inference in semantics: a study of sensory verb complements in English, Glossa 10/2 (1976), 200-240.

Kövecses, Zoltán, Metaphor and Emotion, in: Gibbs, Raymond W. (ed.), The Cambridge handbook of Metaphor and Thought, Cambridge, Cambridge University Press, 2008, 380-396.

Lakoff, George, Sorry, I'm Not Myself Today: The Metaphor System for Conceptualizing the Self, in: Fauconnier, Gilles/Sweetser,Eve (edd.): Spaces, Worlds, and Grammar, Chicago, Chicago University Press, 1996, 91-123.

Langacker, Ronald W., Foundations of Cognitive Grammar: Volume II: Descriptive Application. Stanford, Stanford University Press, 1991.

Maldonado, Ricardo, A media voz: problemas conceptuales del clítico se en español, México, Instituto de Investigaciones Filológicas, UNAM, 1999.

Schmid, Hans-Jörg, English Abstract Nouns as Conceptual Shells. From corpus to cognition, Berlin/New York, Mouton de Gruyter, 2000.

Scovel, Tom, A look-see at some verbs of perception, Language Learning 21 (1971), 75-84.

Sweetser, Eve, From etymology to pragmatics. Metaphorical and cultural aspects of semantic structure, Cambridge, CUP, 1990.

Taylor, John, Linguistic Categorization. Prototypes in Linguistic Theory, Oxford, Oxford University Press, 1995.

Taylor, John, Polysemy and the lexicon, in: Kristiansen, Gitte et al. (edd.). Cognitive Linguistics. Current Applications and Future Perspectives, Berlin/New York, Mouton de Gruyter, 2006, 51-80.

Van Valin, Robert D. Jr./Lapolla, Randy J., Syntax: structure, meaning and function, Cambridge, CUP, 1997.

Viberg, Åke. The lexical typological profile of Swedish mental verbs. Languages in Contrast, 5(1) 2005, 121-157.

Willems, Dominique, Regarde voir: les verbes de perception visuelle et la complémentation verbale, in: Eugeen Roegiest/Liliane Tasmowski (edd.), Verbe et phrase dans les langues romanes. Mélanges offerts à Louis Mourin (RG 20), Gent, 1983, 147-158. 